\title{
Atrevernos a imaginar y a tener miedo. Nuestra responsabilidad ante las generaciones futuras a partir del pensamiento de Günther Anders ${ }^{1}$
}

\author{
Dare to Imagine and Be Afraid. \\ Our Responsibility towards Future \\ Generations Based on the Ideas \\ of Günther Anders
}

\author{
Gabriela Macedo Osorio \\ Universidad Nacional Autónoma de México \\ gabriela.macedo.osorio84@gmail.com \\ orcid.org/oooo-0003-0751-4103
}

Resumen: Günther Anders planteaba que el problema de nuestro tiempo consiste en la discrepancia existente entre el enorme poder tecnológico que desplegamos y las capacidades limitadas que tenemos para comprender, controlar y responsabilizarnos de los efectos de ese poder. Ejemplo de ello es la denominada "isla basura" conformada por 79 millones de toneladas de desechos plásticos que flota en el océano Pacífico. ¿Por qué, aun siendo evidentes los peligros que acontecen, no actuamos? Anders explica que hemos perdido la capacidad de sentir miedo ante la magnitud del peligro que se cierne sobre nosotros. Su propuesta se dirige a alentar este temor a la par que incrementamos nuestra capacidad imaginativa para vislumbrar la magnitud en tiempo y espacio de nuestras acciones, y poder responsabilizarnos y actuar como un compromiso ante las generaciones presentes y futuras.

Palabras clave: miedo, crisis ambiental, imaginación, responsabilidad, isla basura

Abstract: Günther Anders stated that the main problem of our times consists in the discrepancy between the enormous technological power we deploy and the limited capabilities we have to understand, control and take responsibility for the effects of that power. An example of this is

1 Este artículo se realizó gracias al apoyo del proyecto PAPIIT IN 401119 "Heteronomías de la justicia: Territorialidades nómadas". 
the "Great Pacific garbage patch" that floats in the Pacific Ocean and is made up of 79 million tons of plastic waste. Now, even though the dangers that occur are evident, why do we not act? Anders explains that we have lost the ability to be afraid of the magnitude of the danger that looms over us. His proposal is aimed at encouraging this fear while increasing our imaginative capacity to glimpse the magnitude of our actions in time and space, and to be responsible and act as a commitment to present and future generations.

Keywords: fear, environmental crisis, imagination, responsability, garbage patch

Recibido: $\quad 18$ de octubre de 2019

Aceptado: 6 de enero de 2020

Podríamos llamarnos a nosotros mismos utopistas invertidos: mientras que los utopistas corrientes son incapaces de producir realmente lo que pueden imaginar, nosotros somos incapaces de imaginar lo que estamos realmente produciendo.

Günther Anders, Tesis para la Era Atómica

"Porque su suerte ya está echada" (Anders 2011: 19) es la frase con la que inicia el filósofo polaco Günther Anders el primer volumen de La obsolescencia del hombre. Sobre el alma en la segunda revolución industrial, al reeditar por quinta ocasión esta obra en $1979 .{ }^{2}$ En ese momento, el filósofo hace referencia a la manera en cómo nosotros, consumidores de la radio y la televisión, podemos "decidir" la forma en la cual desapareceremos del mundo: de manera inmediata y contundente como la explosión de una bomba o de forma vertiginosa y acelerada como en un descenso en trineo.

La amenaza nuclear, a partir de la utilización de la bomba atómica en Hiroshima y Nagasaki, hizo latente para el filósofo que el peligro se posó sobre nuestras cabezas: en cualquier momento era posible que la humanidad entera desapareciera en un instante y/o sobreviviera rodeada de una "atmósfera contaminada de radiación a la cual no le preocupa ninguna frontera” (Anders 2011: 24).

Por esto el filósofo se volvió un activista acérrimo en contra de la bomba atómica y por supuesto del uso de la energía nuclear. Constantemente daba conferencias, hablaba en la radio y escribía en los periódicos alertando a la población civil, con un lenguaje accesible, pero también deliberadamente alar-

2 En 1956 se publica por primera vez este título. 
mista y contundente, sobre los tiempos apocalípticos que se vivían a partir de la creación de la bomba atómica, pues desde ese momento el ser humano era capaz de eliminar no solo cualquier resquicio de vida, sino de autoexterminarse.

El o6 de agosto de 1945, el Día de Hiroshima, una nueva era comenzó: la era en la que en cualquier momento disponemos del poder para transformar cualquier lugar de nuestro planeta, y aun nuestro planeta mismo, en un Hiroshima. Desde ese día somos, al menos de modo negativo, omnipotentes, pero, puesto que, por otra parte, en cualquier momento podemos ser "borrados", también desde ese día somos totalmente impotentes. Cualquiera que sea el tiempo que esta era pueda durar, aun si durara por siempre, esta es "La última edad": porque no existe ninguna posibilidad de que su differentia specifica, la posibilidad de nuestra autoextinción, pueda terminar sino con el final mismo (Anders 2004: 1).

La advertencia no estaba muy alejada de la realidad pues llegado el siglo xxi el peligro no solo continúa siendo nuclear (no olvidemos Fukushima, ocurrido hace apenas ocho años), sino que ahora también se posa en el exceso de aquellos objetos fabricados con hidrocarburos: el plástico y sus efectos han inundado de forma descomunal nuestro aire, agua, tierra y cuerpo, contribuyendo también al cambio climático, como lo explicaré más adelante.

Los envases y recipientes plásticos poco a poco han sustituido al vidrio y al aluminio, ambos reutilizables y reciclables; pues la fácil producción y manipulación de polímeros, además de su bajo costo, han favorecido su utilización masiva en casi todos los productos y empaques en la industria, así como en nuestra vida cotidiana; con lo cual su acumulación y poco cuidado en su desecho ha resultado en una permanente contaminación del planeta. 3

3 México es el segundo consumidor de envases de PET para refrescos en el mundo, y el primero para recipientes de agua embotellada. Día con día son producidas más de nueve mil millones de botellas PET, que representan casi una tercera parte de la basura doméstica generada en el país; de esta manera, anualmente en promedio, 90 millones de botellas de refrescos y agua purificada son lanzadas a las vías públicas, bosques y playas de nuestro país. Es bien sabido que el consumo de refresco es elevado entre los mexicanos ya que, en promedio, cada habitante ingiere 163 litros al año, siendo de PET la gran mayoría de envases utilizados; como consecuencia, se emplean 450 ooo toneladas de este plástico, aproximadamente. En el caso del agua embotellada, en 2014 se consumieron 234 litros por persona, lo que generó 21 millones de botellas de PET al día, de los cuales solo se recicló 20\%. $C f$. Boletín núm. 
Ante esta problemática, dice Anders:

El amenazado es cualquiera, porque todos somos efectivos consumidores, usuarios y víctimas virtuales de las máquinas y de los productos de las máquinas. Digo de los productos, porque hoy el punto esencial no es quién produce, tampoco la manera de producir, ni siquiera cuánto se produce [...] hoy el debate se centra en el producto mismo (Anders 2011: 23-24).

Es de todos conocida la ruta que llevan nuestros desechos una vez que "nos deshacemos" de ellos: algunos serán reciclados, otros quemados, la mayoría quedarán expuestos al aire libre en basureros fuera de la periferia de la ciudad, otros tantos llegarán al mar siendo parte de los ocho millones de toneladas de plástico que llegan al océano cada año.

En 2018 un grupo de científicos de la Escuela de Ciencias y Tecnología del Océano y la Tierra de la Universidad de Hawái en Manoa, comprobaron que la degradación de los plásticos aumenta la generación de gases de efecto invernadero y con ello el calentamiento global. Según el artículo "Production of methane and ethylene from plastic in the environment", publicado recientemente en la revista científica PLOS ONE, el plástico durante su degradación a la intemperie, exponiéndose a la luz solar, 4 libera una gran cantidad de sustancias químicas entre las que destacan el metano y el etileno, dos potentes gases que contribuyen al efecto invernadero. Estos plásticos, en su mayoría polietilenos, continúan emitiendo gases durante un tiempo indeterminado.

Our results show that different types of plastics, that are commonly used and dispersed in the environment worldwide, produce $\mathrm{CH}_{4}$ and $\mathrm{C}_{2} \mathrm{H}_{4}$ under environmental conditions. [...] The release of greenhouse gases from virgin and aged plastic over time indicates that polymers continue to emit gases to the environment for an undetermined period (Royer et al. 2018).5

3416 de la LXIV Legislatura de la Cámara de Diputados. Publicado el 13 de abril de 2017.

4 En ese mismo artículo, los investigadores sostienen que, si bien la emisión de gases de hidrocarburos se ve reforzada por la fotodegradación a causa de la radiación ultravioleta (UV), las pruebas indican que este tipo de radiación no es esencial para el inicio o la producción continua de hidrocarburos; pero una vez iniciada la liberación de gases nocivos por la radiación solar, esta continúa aún en la oscuridad, a un ritmo que depende de la exposición previa a la radiación (Royer et al. 2018).

5 [Nuestros resultados muestran que los diferentes tipos de plásticos, que se usan y dispersan comúnmente en el medio ambiente en todo el mundo, producen $\mathrm{CH}_{4}$ 
En el caso de los "microplásticos", Sarah-Jeanne Royer, autora del artículo, menciona que estos pueden acelerar aún más la producción de gases de invernadero dado que es proporcional el número de microplásticos con la cantidad de gases de hidrocarburo que producen.

The initial shape of the polymer is also a potential factor contributing to the variability in hydrocarbon production because items of the same mass but with different shapes have different surface-to-volume ratios. Small fragments not only have a greater surface-to-volume ratio than larger items, but they also tend to have longer edge lengths relative to their volume. This predicts that in the environment, as plastic particles degrade and become smaller, they will also emit more hydrocarbon gases per unit mass (Royer et al. 2018). ${ }^{6}$

Günther Anders planteaba que el problema de nuestro tiempo, en ese momento, finales del siglo xx, pero que continúa hasta ahora, consiste en la discrepancia existente entre el enorme poder tecnológico que desplegamos y las capacidades limitadas que tenemos para comprender y controlar los efectos de ese poder, y aunque entonces lo que venía a su mente era la creación de la bomba nuclear, hoy en día podemos extender la preocupación y el sentimiento de catástrofe a toda la humanidad, dado que los efectos de largo y diverso alcance que conlleva la excesiva producción de plásticos nos afectan e implican a todos los seres humanos presentes y fututos.

Al final del prólogo a la quinta edición del primer volumen de La obsolescencia del hombre Anders afirma:

Este informe [...] lo escribí cuando muchos de mis eventuales lectores aún no habían visto la resplandeciente luz de nuestro oscuro mundo. Conocerán que la

(metano) y $\mathrm{C}_{2} \mathrm{H}_{4}$ (etileno) en condiciones ambientales. [...] La liberación de gases de efecto invernadero del plástico virgen y envejecido con el tiempo indica que los polímeros continúan emitiendo gases al medio ambiente durante un periodo indeterminado]. (Todas las traducciones ofrecidas en el texto son mías).

6 [La forma inicial del polímero también es un factor potencial que contribuye a la variabilidad en la producción de hidrocarburos porque los objetos de la misma masa pero con diferentes formas tienen diferentes relaciones de superficie según volumen. Los fragmentos pequeños no solo tienen una mayor relación superficie según volumen que los objetos más grandes, sino que también tienden a tener longitudes de borde más largas en relación con su volumen. Esto predice que en el medio ambiente, a medida que las partículas de plástico se degradan y se vuelven más pequeñas, también emitirán más gases de hidrocarburos por unidad de masa] (Royer et al. 2018). 
situación revolucionaria —o, más exactamente, catastrófica- en que han nacido y en que por desgracia están acostumbrados a vivir demasiado a menudo es la situación en que la humanidad tiene la capacidad de autoaniquilarse; que esta capacidad, ciertamente no honrosa, ya estaba ahí antes de su nacimiento y que los deberes que tienen ya eran los de sus padres y abuelos. Concluyo con el ferviente deseo, para ellos y sus descendientes, de que no se cumpla ninguno de mis pronósticos (Anders 2011: 14-15).

La humanidad se encuentra en un terrible estado de ceguera y de inconciencia frente a la habilidad de comprender y de imaginar las terribles consecuencias que devienen de sus creaciones, acrecentando lo que el filósofo llamará el "desfase prometeico", el cual hace referencia a que nuestras facultades cognitivas: pensar, sentir, responsabilizarse, actuar, imaginar, ya no están a la altura del poder tecnológico, esto es, no podemos representarnos lo que posee enormes dimensiones sobrehumanas, y con ello recordar y responder ante los graves efectos de nuestras acciones ocurridas.

Esto sucede porque los efectos de nuestras acciones $\mathrm{u}$ omisiones son tan grandes, o repercuten después de un tiempo considerable, que no alcanzamos a responder y hacer conciencia de ellos, por lo cual las facultades se pierden al ser incapaces de referirse a los mismos objetos, y la conexión entre ellas queda rota de manera definitiva.

El "desnivel prometeico" se muestra a partir de que, como creadores y productores de un mundo artificial, no estamos a la par de la conciencia, responsabilidad y proyección del "Prometeo que hay en nosotros". Ya nos había mostrado Immanuel Kant que nuestra razón es limitada, pero ahora, afirma Anders, no solo la razón, sino también nuestra imaginación, se encuentran reducidas, pues "en comparación con lo que sabemos y podemos producir, podemos imaginar y sentir demasiado poco; que, en cuanto a sentir, somos menores que nosotros mismos" (Anders 2011: 258).

Ejemplo de ello es la llamada "Isla Basura", también conocida como "Continente de Plástico” o The Great Pacific Garbage Patch, la más grande concentración de microplásticos, desechos plásticos, redes y equipo de pesca abandonado, suspendida en medio del mar. Esta isla se ubica en el giro oceánico del Pacífico Norte, su superficie se estima en un área de 1.6 millones de $\mathrm{km}^{2}$ y se calcula que puede contener cerca de 79 millones de toneladas de desechos (Lebreton et al. 2018). 
Around $60 \%$ of the plastic produced is less dense than seawater. When introduced into the marine environment, buoyant plastic can be transported by surface currents and winds, recaptured by coastlines, degraded into smaller pieces by the action of sun, temperature variations, waves and marine life, or lose buoyancy and sink. A portion of these buoyant plastics however, is transported offshore and enters oceanic gyres. A considerable accumulation zone for buoyant plastic was identified in the eastern part of the North Pacific Subtropical Gyre. This area has been described as 'a gyre within a gyre' and commonly referred to as the 'Great Pacific Garbage Patch'. The relatively high concentrations of ocean plastic occurring in this region are mostly attributed to a connection to substantial ocean plastic sources in Asia through the Kuroshio Extension current system as well as intensified fishing activity in the Pacific Ocean (Lebreton et al. 2018).7

Las consecuencias negativas de esta descomunal acumulación de basura van desde el daño a especies marinas que quedan atrapadas entre las redes y los objetos plásticos, hasta la generación de los mencionados microplásticos, producidos por la acción de la marea y la fotodegradación de los residuos que quedan flotando sobre la capa del agua. ${ }^{8}$

7 [Alrededor de 6o\% del plástico producido es menos denso que el agua de mar. Cuando se introduce en el medio marino, el plástico flotante puede ser transportado por las corrientes superficiales y los vientos, recapturado por las costas y degradado en pedazos más pequeños por la acción del sol, las variaciones de temperatura, las olas y la vida marina, o puede perder flotabilidad y hundirse. Sin embargo, una parte de estos plásticos flotantes se transporta en altamar y entra en giros oceánicos. Se identificó una considerable zona de acumulación de plástico flotante en la parte oriental del Giro Subtropical del Pacífico Norte. Esta área se ha descrito como "un giro dentro de un giro" y comúnmente se conoce como el "Great Pacific Garbage Patch”. Las concentraciones relativamente altas de plástico oceánico que se producen en esta región se atribuyen principalmente a una conexión con fuentes sustanciales de plástico oceánico de Asia a través del sistema actual de la Extensión Kuroshio, así como a la actividad pesquera intensificada en el océano Pacífico.]

8 Recientes investigaciones han mostrado que estas micropartículas plásticas han llegado también al océano profundo al ser ingeridas por larvas cuyos desechos son depositados en el fondo del mar. "Las larvas son componentes abundantes de los conjuntos globales de zooplancton; estas regularmente construyen 'casas' de moco para filtrar las partículas del agua circundante y luego abandonan las estructuras cuando se obstruyen. Al realizar experimentos de alimentación in situ con vehículos operados a distancia, mostramos que las larvas gigantes pueden filtrar una gama de partículas microplásticas de la columna de agua, ingerir y luego empaquetar microplásticos en sus gránulos fecales. Los microplásticos también se adhieren fácilmente a sus casas, las cuales se ha demostrado que se hunden rápidamente en el fondo marino, dejando restos de carbono a los ecosistemas bentónicos. Por lo tanto, las larvas gigantes (Bathochordaeus stygius) pueden contribuir al flujo vertical de 
Los polímeros se fragmentan cada vez más llegando a niveles microscópicos, que se esparcen a través de las corrientes marinas abarcando extensiones mayores de agua, arena, arrecifes y también de animales, pues estos los consumen junto con el plancton del cual se alimentan, lo que hace que el plástico entre a la cadena alimenticia marina y humana. 9

¿Por qué, aun siendo evidentes los peligros que acontecen al mundo entero, no actuamos? Anders explica que los seres humanos hemos perdido la capacidad de sentir miedo ante la magnitud del peligro que se cierne sobre nosotros. "Vivimos en la época de la incapacidad para el miedo" (Anders 2011: 253), definiéndolo como el sentimiento de angustia ante la proximidad de algún daño real o imaginario.

El miedo, escribirá el filósofo y urbanista francés Paul Virilio, está mal visto, es "señal de un temperamento débil y de una falta de consistencia personal" (Virilio 2012: 8). Hoy en día esa concepción ha tenido que cambiar pues en un mundo global, en el que las nuevas tecnologías han acortado el tiempo y el espacio acercándonos en "tiempo real" con "todo el mundo", los Estados tienen que ser capaces de convencer a los ciudadanos de que aún pueden preservar su seguridad física.

El miedo hoy en día es un entorno, un medio, un mundo. Nos ocupa y nos preocupa. Antes, el miedo era un fenómeno relacionado con acontecimientos localizables, identificables y circunscritos en el tiempo: guerras, hambrunas, epidemias [...]. Hoy es el mundo mismo, limitado, saturado, encogido lo que nos oprime y nos "estresa" provocando una especie de claustrofobia: crisis bursátiles contaminantes, terrorismo indiferenciado, pandemias fulgurantes, suicidios "profesionales". El miedo es mundo, es pánico en el sentido del término griego, en el sentido de "totalidad" (Virilio 2012: 16).

microplásticos a través del rápido hundimiento de los gránulos fecales y las casas desechadas. Las larvas, y potencialmente otros alimentadores de filtros pelágicos abundantes, pueden comprender un nuevo mecanismo de transporte biológico que entrega microplásticos desde las aguas superficiales, a través de la columna de agua, al fondo marino" (Katija y Sherlock 2017).

9 "Los microplásticos son el $94 \%$ de los 1.8 billones de fragmentos de plástico de la mancha. Pero eso solo equivale al ocho por ciento del tonelaje total. Resulta que, de las 79 ooo toneladas métricas de plástico de la mancha, la mayoría son equipos de pesca abandonados, no las botellas de plástico o los embalajes que llenan los titulares cuando se trata este tema" (Parker 2018). 
Las sociedades viven bajo el influjo de una fascinación deslumbrante por el poder tecnológico que día a día constituye una fuente de riesgo para el porvenir de la existencia humana. Se confía en la omnipotencia de las creaciones humanas y en nuestra capacidad para generarlas, innovarlas y perfeccionarlas, pero ese poder también va de la mano con la capacidad de autodestruirnos. Ya Anders afirmaba que:

Si en la conciencia del hombre actual hay algo que se considera absoluto o infinito, ya no es el poder de Dios, tampoco el poder de la naturaleza, por no hablar de los supuestos poderes de la moral o la cultura, sino nuestro poder. En lugar de la creatio ex nihilo, que manifiesta omnipotencia, ha aparecido su contrapoder: la potestas annihilationis, la reductio ad nihil; un poder que está en nuestra propia mano. La omnipotencia prometeica, anhelada desde tanto tiempo, se ha hecho en verdad nuestra, aunque de forma diferente a la esperada. Dado que poseemos el poder de ponernos fin unos a otros, somos los señores del apocalipsis. Lo infinito somos nosotros (Anders 2011: 230-231).

Este poder está ligado a intereses militares, políticos y económicos; la elaboración y utilización de la ciencia y la tecnología en estrategias bélicas es cada vez mayor; tal es el caso del uso de drones, la localización por GPS, las armas bacteriológicas y de destrucción masiva, entre muchas más. La ciencia, menciona Paul Virilio en su texto La administración del miedo:

Empieza a militarizarse a partir de la guerra de 1914 con la utilización del gas en el campo de batalla, y no lo hace enteramente hasta la bomba $\mathrm{H}$, que corresponde a otro nivel puesto que se trata de un arma absoluta. [...] A partir de Hiroshima, las democracias occidentales, la URss y luego Rusia, el resto del mundo a través de alianzas y los acuerdos diplomáticos, vivimos bajo un régimen militar que domina la vida política [...] si hemos evitado la guerra atómica, es más por un milagro de la historia que por las supuestas virtudes de la disuasión mutua (Virilio 2012: 25-26).

Es por ello que la invitación de Anders a atreverse a tener miedo y con ello ampliar los límites de nuestra imaginación van ligados, pues en la medida en que nos permitimos imaginar ampliamente las consecuencias negativas de las acciones que realizamos - las cuales tendrán una repercusión más allá del tiempo que vivamos y el lugar en el que las desarrollemos— será posible 
extender el miedo por aquello que puede suceder y que está ocurriendo si no se pone un alto o se deja de actuar tal como se ha venido haciendo.

Este temor nos llevará a denunciar, protestar, increpar a las autoridades y las empresas, a hacernos responsables por la sobrevivencia y bienestar de las generaciones venideras.

Pero ¿qué implica ampliar los límites de vuestra imaginación? ¿Por qué la facultad imaginativa se ve ligada en Anders con aspectos morales como la responsabilidad, la concientización, el compromiso, etc.?

Una de las definiciones del concepto de imaginación propuesto por la Enciclopedia de Filosofía de Stanford nos dice que es la capacidad de "to imagine is to represent without aiming at things as they actually, presently, and subjectively are. One can use imagination to represent possibilities other than the actual, to represent times other than the present, and to represent perspectives other than one's own”10 (Liao y Gendler 2019). Esto apuntaría a que es posible "traer al presente" situaciones que aún no han ocurrido, proyectando en ellas probabilidades que pudieran acontecer dada la información y evidencia tenida.

Esta facultad se distingue de percibir y creer, dirán más adelante los autores de esta entrada, porque "Unlike perceiving and believing, imagining something does not require one to consider that something to be the case. Unlike desiring or anticipating, imagining something does not require one to wish or expect that something to be the case"11 (Liao y Gendler 2019), mientras en los anteriores estados sí se requiere que aquello percibido, recordado o creído ciertamente ocurra o se haya suscitado.

En sus “Tesis para la Era Atómica”, Anders profundiza en la importancia de ampliar la imaginación en contraste con la incapacidad humana para sentir, imaginar o arrepentirse por la eliminación de 100 ooo semejantes, pues este número sobrepasa nuestro poder imaginativo; es este "desnivel prometeico" descrito con anterioridad, en el que "entre más grande es el abismo, tanto más débil es el mecanismo de frenaje" (Anders 2004: 4). A esta característica la llama supraliminal y la describe como una situación en la cual "el estímulo

10 Representar sin apuntar a las cosas como son en realidad, presente y subjetivamente. Se puede usar la imaginación para representar posibilidades distintas de la actual, para representar momentos distintos del presente y para representar perspectivas distintas de la propia.

11 Imaginar algo no requiere que uno considere que ese es el caso. A diferencia de desear o anticipar, imaginar algo no requiere que uno desee o espere que ese sea el caso. 
es demasiado grande como para generar una reacción, o para activar algún mecanismo de frenaje" (Anders 2004: 4).

A diferencia de los sentidos y la percepción, Anders considera que la imaginación puede llevar al ser humano a la verdad, pues mientras los primeros son a su juicio miopes y su horizonte es "sensiblemente estrecho", a través de la imaginación el ser humano puede ampliar su horizonte y "tratar de visualizar ese infinito, aunque al tratar de hacerlo violente la 'estrechez natural' de su imaginación" (Anders 2004: 4).

Con lo cual, una imaginación moral implicaría poder representarnos a través de las emociones y sentimientos aquello que es distante en tiempo y espacio. Permitiría transformar y ampliar la relación entre nuestra capacidad humana de proyectar y empatizar con la magnitud y la escala de los hechos; es decir, si para Anders hay "volúmenes" y distancias temporales y espaciales que al ser humano le son imposibles de vislumbrar a causa del "desnivel prometeico", ampliar la imaginación moral daría la posibilidad de llorar a los miles de muertos, que, aunque todavía no fallecen, ya están sentenciados a este fatídico destino.

Cada facultad tiene sus límites de competencia, más allá de los cuales ya no funciona y, por tanto, no puede registrar aumentos; los ámbitos de las facultades no concuerdan. [...] "podemos asesinar a miles; tal vez podemos imaginar a diez muertos; pero a lo sumo solo podemos lamentarnos o deplorar por uno" (Anders 2011: 256).

Para Hannah Arendt, primera esposa de Günther Anders, la imaginación tiene un componente conciliador y orientador ya que puede "colmar el abismo que nos separa de aquello que está demasiado lejos y verlo como si nos fuera familiar" (Arendt 1995: 45). En su texto "Comprensión y política”, la filósofa afirma, frente a las atrocidades acaecidas en el siglo Xx, haciendo especial referencia a los totalitarismos, que estos han destruido el sentido común y el juicio; sin embargo, el hombre, como ser que "actúa", puede reconciliarse con la realidad a través de la facultad de la imaginación ligada con la comprensión. "Sin este tipo de imaginación que en realidad es la comprensión, no seríamos capaces de orientarnos en el mundo. Es la única brújula interna de la que disponemos" (Arendt 1995: 46).

El que la imaginación sea una especie de "brújula interna" supone que el ser humano puede orientarse hacia el polo que lo dirija al discernimiento de lo correcto; mientras que "comprender" indicaría entender a aquellos que 
han actuado de forma totalitaria. Implicaría situarse entre dos bandos: las víctimas y los victimarios, pero para Anders estas distinciones se han borrado, pues si bien es posible identificar a aquellos que amenazan la estabilidad y la vida, a quienes sin duda se debe combatir, ${ }^{12}$ una vez que el fin llegue, todos (víctimas y victimarios) perecemos por igual. ${ }^{13}$

En el texto "Humanos y terrícolas en la Guerra de Gaia", Deborah Danowski y Eduardo Viveiros de Castro explican que el concepto 'humanidad', usado como término universal, no puede subsumir positivamente de los particulares sino solo en el aspecto negativo, es decir, cuando resulta inminente la llegada de un apocalipsis desnudo. Se preguntan los autores si esto significa que la única finalidad común de la humanidad es su fin, su extinción (Danowski y Viveiros de Castro 2019: 156).

La respuesta es afirmativa y en concordancia con el filósofo polaco, pues el advenimiento de la era nuclear causó una mutación en la noción de especie: "pasamos de la condición de un 'género de mortales' a la de un 'género mortal', una especie cuyo fin se tornó metafísicamente inminente" (Danowski y Viveiros de Castro 2019: 156).

Pensar, como mencionamos en los párrafos anteriores, que existen de forma separada el grupo de víctimas y el de victimarios resulta equivocado, pues sugeriría que la humanidad tendría la posibilidad de cometer o no el acto de destrucción masiva, que la esperanza puede estar depositada en la voluntad de los que toman la decisión. Pero Anders piensa de forma contraria, pues no tenemos derecho a encubrir la existencia de dos lados distintos e irreconciliables, el de los culpables y los inocentes, pues la catástrofe no conservará ninguna dualidad; ambos grupos perecerán; "solo habrá una

12 Danoswski y Viveiros de Castro están de acuerdo con Anders en que: "si como en el apocalipsis nuclear, todos acabaremos, unos antes que otros, siendo víctimas de la ruptura de los planetary boundaries, eso no impide que se puedan y deban identificar los lados enemigos [...] ya escribía el propio Anders: El tiempo del fin en que vivimos... contiene dos tipos de hombres: los culpables y las víctimas. Debemos tener en cuenta esa dualidad en nuestra reacción: nuestra tarea tiene por nombre 'combate'" (Danowski y Viveiros de Castro 2019: 158).

13 Aunque si bien es cierto que los países más desarrollados e industrializados son aquellos que más contribuyen al calentamiento global, son también los "menos vulnerables" en el sentido de que la desigualdad económica que a ellos les favorece, los ayuda a mitigar los efectos devastadores del cambio climático en su territorio. Tenemos el caso de los Estados Unidos de Norteamérica que, si bien firmó el Acuerdo de París en 2015 comprometiéndose a reducir su emisión de gases, en 2017 su presidente recién electo Donald Trump, anunció su salida del Acuerdo ( $c f$. Pereda 2017). 
humanidad cuando ya no haya humanidad alguna, cuando desaparezca el último ser humano de la faz de la tierra" (Danowski y Viveiros de Castro 2019: 157).

La imaginación y el temor se cruzan cuando Anders nos invita a "imaginar la nada", pues el temor "es la imaginación de la nada en concreto" (Anders 2004: 5). Debemos temer, estamos obligados a temer, porque nuestra capacidad de tener miedo es demasiado pequeña y no corresponde a la magnitud del peligro actual, que consiste en llegar a un punto en el que la nada sea la constante de lo que un día fue nuestro mundo.

Por eso nuestro imperativo debe ser: "expande la capacidad de tu imaginación, [lo cual] significa en concreto, incrementa tu capacidad de temer. Por tanto, no temas temer; ten el coraje de estar aterrorizado, y de aterrorizar a los demás" (Anders 2004: 5). Entre más grande sea el efecto posible de nuestras acciones, debemos ampliar más nuestra capacidad imaginativa y de respuesta para poder representárnoslo y hacer algo al respecto.

Frente a esto, aclama Anders, "la única tarea moral decisiva de ahora consiste en educar la imaginación moral” (Anders 1995: 80), lo cual implica ajustar, ampliar e incluso exagerar en la proyección de nuestra imaginación para poder acercarnos a la desproporción inimaginada de nuestras propias creaciones.

En este punto también convergen Virgilio y Anders, pues para el filósofo francés:

Solo de la conciencia más profunda de la crisis surgirá una esperanza política. De ahí mi deseo de una "universalidad del desastre" y de una comprensión colectiva de los límites. Hemos alcanzado los límites en el sentido de que la Tierra es demasiado pequeña y el mundo está forcluido. ${ }^{14} \mathrm{Si}$ hay alguna esperanza, esta reside en una comprensión colectiva y universal de la situación real que vive el mundo (Virilio 2012: 66).

Si pudiéramos imaginar un mundo en el que la humanidad, así como todo lo que nos rodea, ya no existiera más, es decir, desapareciera, aniquilada por

14 Paul Virilio en una nota a pie de página explica que este neologismo expresa "el cambio que se ha producido en las sociedades avanzadas que han pasado de la ciudad como espacio de libertad a ciudades privadas protegidas por su cerco eléctrico, cámaras de seguridad y guardias: son síntomas de la regresión patológica de la ciudad, según la cual la cosmópolis, la ciudad abierta de ayer cede lugar a esta claustrópolis" (Virilio 2012: 52). 
las acciones que hacemos o por aquellas que no logramos transformar, realmente, más de una vez pensaríamos en realizar o no tal acción.

Con lo cual, si consideramos que el futuro "ya no viene" sino que "lo hacemos", también está inscrita la posibilidad de su interrupción. "Tenemos, pues, poder sobre un tiempo, que habitualmente no tomamos ni podemos tomar en consideración como 'futuro'. Nuestra acción va más allá de nuestra comprensión. Lanzamos más allá de lo que, miopes, podemos ver" (Anders 2011: 217).

Es importante que entendamos que la responsabilidad de nuestros actos no termina en el ahora, sino que se extiende a las generaciones venideras pues los productos y artefactos que creamos, y con ello sus desechos y repercusiones materiales y espaciales, tienen un rango mayor de subsistencia que el de hace 100 años; con lo cual somos responsables también por los aún no nacidos. "Ya lo decía en un relato molúsico:15 'Saluda a los no nacidos como si fueran tus vecinos' [...] [pues] lo que se exige no es, por ejemplo, prever a la manera de los profetas esto o aquello, sino únicamente de que tratemos de comprender el horizonte ampliado como nuestro" (Anders 2011: 271).

Resulta prioritario que este mundo tecnológico que el ser humano ha creado se transforme y sea impulsado por personas que tengan en la mira el fin de nuestros tiempos y se atrevan a tener miedo por ello, a expresarlo y contagiarlo. Personas conscientes de la vulnerabilidad ambiental y humana que "vean en ella un escándalo; para socavar los límites 'bien establecidos' e inamovibles; para convertirlos en obstáculos que hay que salvar; para hacer retroceder nuestra esquizofrenia" (Anders 2007: 77).

Un ejemplo de ello se materializa en la joven activista medioambiental sueca Greta Thunberg ${ }^{16}$ y su movimiento Fridays For Future, el cual busca

15 En 1931, Günther Anders comienza a escribir una novela llamada La catacumba molúsica, en la cual retrata los regímenes fascistas y sus aparatos de propaganda y adoctrinamiento en la ciudad de Molusia.

16 Greta Tintin Eleonora Ernman Thunberg nació en Estocolmo en 2003. En agosto de 2018 comenzó a manifestarse frente al parlamento sueco, para que este tomara medidas más contundentes contra el cambio climático. Cada viernes, desde ese mometo hasta las elecciones generales de Suecia en septiembre del mismo año, Greta faltaba a la escuela y se paraba frente al parlamento sosteniendo un cartel que decía: "Huelga escolar por el clima" (SKOLSTREJK FÖR KLIMATET). En su demanda exigía que el gobierno sueco redujera las emisiones de carbono con base en lo establecido en el Acuerdo de París, firmado en 2016.

Participó en la manifestación Rise for Climate (En Pie por el Clima) en las afueras del Parlamento Europeo en Bruselas y en la Declaración de la Rebelión organizada por el movimiento Extinction Rebellion en Londres. El 4 de diciembre de 2018, Thunberg ofreció un discurso en la Conferencia de las Naciones Unidas sobre el Cambio Climático 
generar conciencia sobre las causas y los efectos del cambio climático. ${ }^{17}$ En su discurso durante la Asamblea Anual del Foro Económico Mundial celebrado en Davos, en enero de 2019, Greta vuelve a hacer el llamado de Anders: tener el valor de tener miedo, porque "nuestra casa está en llamas"; el planeta arde por nuestra descomunal generación y emisión de dióxido de carbono.

Este primer paso, atreverse a temer, es esencial para poder ser conscientes de que el peligro es real, tangible, inmediato; pues, así como Anders buscaba alertar del peligro latente por el uso de la energía nuclear, Greta continúa la propagación de este pánico activo al increpar: "No quiero que tengas esperanzas. Quiero que entres en pánico. Quiero que sientas el miedo que siento todos los días. Y luego quiero que actúes. [...] Quiero que actúes como lo harías en una crisis. Quiero que actúes como si nuestra casa estuviera en llamas. Porque lo está" (Thunberg 2019).

El planeta, y las formas de vida en él, ya no pueden soportar un sistema de producción y consumo como el que hemos venido realizando a lo largo del siglo xx. Gobiernos, industrias y ciudadanía debemos reconocer que, afirma Greta, "estamos fallando, pero todavía hay tiempo para cambiarlo todo. Aún podemos arreglar esto. Todavía tenemos todo en nuestras propias manos. Pero a menos que reconozcamos las fallas generales de nuestros sistemas actuales, lo más probable es que no tengamos una oportunidad" (Thunberg 2019).

Debemos encontrar una nueva forma de relacionarnos con el futuro, una nueva modalidad de "conocimiento moral imaginativo", más que cognoscitivo, para poder cambiar el rumbo y convertir al porvenir en objeto de nuestra responsabilidad.

de 2018 (COP24), celebrado en Polonia. Este discurso obtuvo gran notoriedad en numerosos medios de comunicación alrededor del mundo, lo cual la puso en la mira tanto de niños, jóvenes y activistas que se suman a su lucha, como de adversarios y enemigos políticos y sociales que buscan desacreditarla.

17 Este movimiento ambientalista juvenil ha crecido exponencialmente. Sus simpatizantes exigen que los gobiernos, sobre todo de países industrializados, respeten los Acuerdos de París en los que se comprometieron a disminuir sus emisiones de $\mathrm{CO}_{2}$, y con ello reducir $2^{\circ} \mathrm{C}$ la temperatura del planeta. La primera huelga global por el clima, celebrada el 15 de marzo de 2019, congregó a más de dos millones de personas en 2416 ciudades del mundo. Mientras que la cuarta huelga global por el clima, ocurrida el 29 de noviembre de ese mismo año, en vísperas de la Conferencia de las Naciones Unidas sobre el Cambio Climático en Madrid, se expandió por 2 558 ciudades y reunió a más de un millón de personas de todos los continentes ( $c f$. $<$ https://www.fridaysforfuture.org/statistics/list-towns $>$, consultado por última vez el 8 de diciembre de 2019 . 
Salvar el abismo existente entre tu capacidad de hacer y de imaginar; reducir el desnivel existente entre ambas capacidades; o dicho de otro modo: debes ampliar considerablemente el limitado ‘ámbito de acción' de tu imaginación (y de tu capacidad de sentir, todavía más limitada), hasta que imaginación y sentimiento puedan captar y comprender la magnitud de lo que eres capaz de hacer; hasta que seas capaz de aceptar lo captado, o de rechazarlo (Anders 2007: 77-78).

Si se continúa evadiendo el desastre, si continuamos negando las evidencias que muestran que el fin está cerca, que el cambio climático es real y está sucediendo tal como lo denuncia Greta Thunberg; que nuestra producción y consumo de plásticos es descomunal, ya que nuestros productos y sus efectos sobrepasan no solamente nuestra capacidad de visualizar o sentir, sino también la medida de lo que somos capaces de usar, ${ }^{18}$ nunca seremos capaces de sentir temor, de creer que el Apocalipsis es ahora, y esta ceguera impedirá ampliar la imaginación moral y actuar para cambiar las cosas. Hay que atreverse a tener miedo para dimensionar el desastre, pero sobre todo para actuar de forma inmediata.

Si no tenemos en la mente, tercamente, la gran probabilidad del desastre, y si no actuamos consecuentemente, entonces seremos incapaces de encontrar una salida, No hay nada más aterrorizador que estar en lo correcto. Y si algunos, paralizados por la lóbrega probabilidad de la catástrofe, ya han perdido el coraje, todavía tienen una oportunidad de probar su amor por el hombre, haciendo caso de la máxima cínica: "Sigamos trabajando como si tuviéramos derecho a esperar. Nuestra desesperación no nos concierne" (Anders 2004: 10).

\section{Bibliografía}

ANDERS, Günther (1995). Llámese cobardía a esa esperanza. Bilbao, Besatari.

Anders, Günther (2004). “Tesis para la era atómica”, Revista Artefacto. Pensamientos sobre la técnica, núm. 5: 1-11.

ANDERS, Günther (2007). "Mandamientos de la era atómica”, Filosofía de la situación. Madrid, Catarata: 75-86.

ANDERS, Günther (2011). La obsolescencia del hombre. Sobre el alma en la época de la segunda revolución industrial, vol. 1. Valencia, Pre-Textos.

18 Anders da cuenta de que "hoy en día hemos alcanzado la situación en la que se manufacturan productos que simplemente no pueden ser necesitados, y que son demasiado grandes en un sentido absoluto" (Anders 2004: 6). 
ArendT, Hannah (1995). “Comprensión y política”, De la historia a la acción. Barcelona, Paidós: 29-46.

Cámara de Diputados (2017). Boletín núm. 3416 de la LXIV Legislatura de la Cámara de Diputados, en <http://www5.diputados.gob.mx/index.php/esl/Comunicacion/ Boletines/2017/Abril/13/3469-En-Mexico-9o-millones-de-botellas-de-plasticode-refrescos-y-agua-son-lanzados-a-la-via-publica-rios-y-mares $>$, consultado por última vez el 3 de septiembre de 2019.

Danowski, Deborah, y Eduardo Viveiros de Castro (2019). "Humanos y terrícolas en la Guerra de Gaia”, ¿Hay mundo por venir? Ensayo sobre los miedos y los fines. Buenos Aires, Caja Negra: 149-196.

Katija, Kakani, y Rob E. Sherlock (2017). "From the surface to the seafloor: How giant larvaceans transport microplastics into the deep sea”, Science Advances, vol. 3, núm. 8, en <https://advances.sciencemag.org/content/3/8/e1700715>, consultado por última vez el 7 de agosto de 2019.

Lebreton, L., Boyan Slat, F. Ferrari, et al. (2018). "Evidence that the Great Pacific Garbage Patch is rapidly accumulating”, Scientific Reports, 8, en <https://www. nature.com/articles/s41598-018-22939-w>, consultado el por última vez el 7 de agosto de 2019 .

LiaO, Shen-yi, y Tamar Gendler (2019). “Imagination”, Stanford Encyclopedia of Philosophy, en <https://plato.stanford.edu/entries/imagination/>, consultado por última vez el 8 de diciembre de 2019.

PARKER, Laura (2018). “¿De qué está hecha la isla de basura en el pacífico?”, en <https:// www.nationalgeographic.es/medio-ambiente/2018/o3/de-que-esta-hecha-la-islade-basura-del-pacifico>, consultado por última vez el 7 de agosto de 2019.

Pereda, Cristina (2017). "Qué ocurre con el Acuerdo de París tras el abandono de Estados Unidos”, en <https://elpais.com/internacional/2017/05/31/estados_unidos/1496238308_555328.html>, consultado porúltima vez el 9 de diciembre de 2019.

Royer, Sarah-Jeanne, Sara Ferrón, Samuel T. Wilson, David M. Karl (2018). "Production of methane and ethylene from plastic in the environment", PLOS ONE en $<$ https://doi.org/10.1371/journal.pone.0200574>, consultado por última vez el 8 de diciembre de 2019.

Thunberg, Gretta (2019). "Our house is on fire", en <https://www.theguardian.com/ environment/2019/jan/25/our-house-is-on-fire-greta-thunberg16-urges-leadersto-act-on-climate>, consultado por última vez el 4 de septiembre de 2019.

Virilio, Paul (2012). La administración del miedo. Madrid, Pasos Perdidos/Barataria. \#Fridaysforfuture (2019). "Statistics/List-towns", en <https://www.fridaysforfuture. org/statistics/list-towns>, consultado por última vez el 8 de diciembre de 2019.

\section{Gabriela Macedo Osorio}

Candidata a doctora en Filosofía por la UNAM. Entre sus artículos recientes destacan: "La bomba nuclear y nuestra ceguera ante el Apocalipsis. Una reflexión sobre la responsabilidad temporal y espacial de nuestras acciones a partir de 
Günther Anders”, en Revista Intersticios. Filosofía, Arte, Religión (2018); "Hacia una reflexión sobre la crisis ambiental. Max Horkheimer y Günther Anders. Afán de dominio y desfase prometeico", en Bajo palabra. Revista de Filosofía. Monográfico: La teoría crítica de una orilla a otra (2019) y "El 'desfase prometeico' y la 'vergüenza prometeica' en Günther Anders. Una forma de expresión de la naturaleza contingente del ser humano”, en el libro Heteronomías de la justicia: de exilios y utopías (2019). 